Revue d'histoire de l'Amérique française

REVUE D.HISTOIRE DE L'AMÉRIQUE FRANÇAISE

\title{
Comment dénombrer les serviteurs de l'État au Canada-Uni. Essai méthodologique
}

\section{Bruce Curtis}

Volume 46, numéro 4, printemps 1993

URI : https://id.erudit.org/iderudit/305147ar

DOI : https://doi.org/10.7202/305147ar

Aller au sommaire du numéro

Éditeur(s)

Institut d'histoire de l'Amérique française

ISSN

0035-2357 (imprimé)

1492-1383 (numérique)

Découvrir la revue

Citer cet article

Curtis, B. (1993). Comment dénombrer les serviteurs de l'État au Canada-Uni. Essai méthodologique. Revue d'histoire de l'Amérique française, 46(4), 607-628. https://doi.org/10.7202/305147ar
Résumé de l'article

Inscrit dans le débat sur la " nineteenth century revolution in government ", cet article propose des considérations méthodologiques pour identifier et dénombrer le personnel de l'État au Canada-Uni. Après avoir relevé les embûches des sources officielles, l'article souligne les limites méthodologiques du modèle bureaucratique du pouvoir de l'État. 


\title{
COMMENT DÉNOMBRER LES SERVITEURS DE L'ÉTAT AU CANADA-UNI ESSAI MÉTHODOLOGIQUE ${ }^{1}$
}

\author{
BRUCE CURTIS \\ Département de sociologie et d'anthropologie \\ Université Wilfrid Laurier
}

\section{RÉSUMÉ}

Inscrit dans le débat sur la «nineteenth century revolution in government», cet article propose des considérations méthodologiques pour identifier et dénombrer le personnel de l'État au Canada-Uni. Après avoir relevé les embûches des sources officielles, l'article souligne les limites méthodologiques du modèle bureaucratique du pouvoir de l'État.

\begin{abstract}
In keeping with recent contributions to the debate over the "nineteenth century revolution in government", this article proposes methodological considerations for the identification and enumeration of servants of the state in the United Canadas. After an examination of difficulties presented by the use of official source materials, the article highlights the methodological limitations of a bureaucratic model of state power.
\end{abstract}

Les grandes interventions politiques et les innovations administratives importantes de la période 1841-1867 ont mené à la prolifération du personnel de l'État au Canada-Uni. Pour le gouvernement central, dans ce que l'on appelle l' «inside service» (ceux qui travaillent au siège du gouvernement), on dénombre 86 membres en 1841 et 114 en 1846 . Cette partie de la bureaucratie étatique compte 260 employés salariés en 1860, et 317 en 1863. Au département des

1. Ce travail a été rendu possible grâce à une subvention du Conseil de recherches en sciences humaines du Canada et grâce au soutien de l'Université Wilfrid Laurier. Je tiens à remercier Michèle Martin, Jean-Marie Fecteau, Patricia Kennedy et Michael Piva, en plus des quatre lecteurs de la Revue d'histoire de l'Amérique française. 
postes, le personnel aurait presque quadruplé entre 1851 et $1862^{2}$. Les dépenses totales du gouvernement colonial, qui sont d'environ $2040000 \$$ en 1846, dépassent légèrement $12800000 \$$ en 1864-1865. Pourtant, aucune analyse systématique du développement du personnel de l'État n'existe encore. Cet article propose des considérations méthodologiques pour le dénombrement des membres du personnel étatique au Canada-Uni ${ }^{3}$.

À première vue, retracer la croissance du nombre des employés paraît chose facile. Les sources documentaires pour une telle reconstruction au cours de la période de l'Union des Canadas, sont multiples et faciles d'accès. Parmi les nombreux comptes rendus plus ou moins détaillés du personnel payé directement par le gouvernement, nous notons la comptabilité publique, les listes de mandats émis par le receveur général, le «Blue Book», les listes de paie des départements et, depuis la fin des années 1850, la main courante du vérificateur

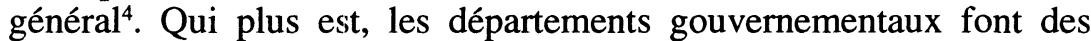
rapports au Parlement tous les ans, rapports souvent très complets et normalement publiés comme «documents parlementaires». De telles ressources devraient permettre le dénombrement du personnel de l'État, et leur caractère multiple devrait offrir un moyen de contrôler l'exactitude d'un tel effort. Pourtant, la tâche n'est pas aussi facile qu'elle peut paraître. L'intérêt que nous portons à la croissance du nombre des travailleurs de l'État se situe dans la perspective des vues de Corrigan et Sayer sur la «nineteenth century revolution in government». Afin de mieux comprendre la montée de l'État bourgeois, ces auteurs font la distinction, à la suite de Philip Abrams, entre le «state system», comprenant les structures et les institutions législatives, exécutives, juridiques, et administratives, et le «state project». Ce dernier concept, chez Corrigan et Sayer, sert à analyser l'État comme le lieu de continuelles tentatives d'établir et de justifier les hégé-

2. Archives nationales du Canada [ANC], RG1 E13, le Blue Book, 1841 et 1846; département des finances, bureau du vérificateur, RG19 vol. 2083, 30 juin 1860, vol. 2089, 31 décembre 1863. Pour les postes, voir le rapport de Thomas D'Arcy McGee sur l'organisation gouvernementale, un document fondamental, ANC, RG1 E7 vol. 59A.

3. Ces chiffres sont tirés de la comptabilité publique annuelle et ne tiennent pas compte de la possibilité d'inflation. Voir les Documents parlementaires, 1841-1866. Notons le travail en cours de Michael Piva sur la bureaucratie canadienne; voir, par exemple, «Anatomy of a Bureaucracy: the Civil Service Act of 1857 Reconsidered», communication inédite, département d'histoire, Université d'Ottawa, 1992.

4. Le Blue Book est un rapport statistique annuel livré au gouvernement impérial par le gouverneur général. Nous ne parlerons pas des listes de paie départementales dans cet article. Pour les reconstituer, il faudrait examiner la correspondance de tous les départements, une tâche qui est, pour le moment du rnoins, au-delà de nos moyens. La main courante du vérificateur donne un résumé très complet des membres de la bureaucratie, mais, malheureusement, seulement pour les années 1860-1863. Voir ANC RG19, vols. 2083-2089. 
monies sociales par des moyens administratifs, légaux, financiers et symboliques plus ou moins violents. Les initiatives ou projets étatiques se fondent sur les structures et les institutions du state system, mais mobilisent aussi un personnel qui leur est propre ${ }^{5}$.

Le state system, considéré du point de vue du personnel impliqué, pourrait se rapprocher des conceptions courantes de la fonction publique. Le state project, en tant qu'ensemble des projets hégémoniques des groupes qui investissent l'État, est une entité plus ou moins stable qui fluctue selon les contestations dont il est l'objet. Il est donc difficile d'en évaluer rigoureusement le personnel. Pourtant, si nous pouvions dénombrer les gens mobilisés dans les projets et les initiatives de l'État, nous comprendrions mieux le processus de son développement au Canada-Uni.

Selon Corrigan et Sayer, la formation de l'État bourgeois implique l'émergence d'une tension dynamique entre la vie privée et la vie publique, entre la société civile et l'État. Les projets de régulation venant de l'État portent atteinte à la vie privée, et les formes et les pratiques de régulation venant de la société civile menaçent l'ordre politique. Cette tension dynamique est l'un des moteurs du développement des structures et des initiatives étatiques ${ }^{6}$.

Chez ces auteurs, la compréhension de la formation de l'État passe non seulement par la reconstitution du développement du state system, mais exige également la connaissance du state project. Il s'agit de découvrir, de retracer et d'évaluer dans le mode de régulation étatique, l'encadrement de la vie privée et de la conscience populaire. Dans leur optique, lorsque le mode de régulation étatique réussit à pénétrer jusqu'au cœur de la vie privée, «l'État» devient l'état dans lequel la population se trouve, ou encore la condition sociale; lorsque les hégémonies sociales passent entièrement par l'État, la population

5. Pour l'élaboration de ces concepts, Philip Abrams, «Notes on the Difficulty of Studying the State», Journal of Historical Sociology, 1,1 (1988): 58-89; et Philip Corrigan et Derek Sayer, The Great Arch: English State Formation as Cultural Revolution (Oxford, Basil Blackwell, 1985). Chez Abrams, cette distinction a des finalités essentiellement heuristiques; il ne faut pas prendre au pied de la lettre sa suggestion que l'État n'existe pas; si l'espace nous permettait de nuancer, nous parlerions de la relation dialectique entre state system et state project.

6. La régulation des formes d'association au $\mathrm{XIX}^{\mathrm{e}}$ siècle est un exemple de cette dynamique; voir Jean-Marie Fecteau, «État et associationnisme au XIX ${ }^{\mathrm{e}}$ siècle québecois: éléments pour une problématique des rapports États/société dans la transition au capitalisme», A. Greer et I. Radforth, eds., Colonial Leviathan: State Formation in Mid-Nineteenth-Century Canada (Toronto, University of Toronto Press, 1992), 134-162; Bruce Curtis, «Preconditions of the Canadian State: Educational Reform and the Construction of a Public in Upper Canada, 1837-1846», Studies in Political Economy, 10 (1983): 99-121. 
vit l'État comme voix, désir, et compréhension. L'identité du sujet politique incarne l'État ainsi conçu.

Cette prise de position sur l'État a été critiquée par Valverde et Weir, qui insistent néanmoins sur l'importance de concevoir l'État comme un processus politique, et sur la nécessité de reconnaître l'aspect culturel, au sens large, du mode de régulation étatique. Mais, suggèrent-elles, Corrigan et Sayer risquent de faire disparaître l'État en l'assimilant à la société civile. Si l'État est partout, il n'est nulle part; il cesse d'être accessible comme objet d'analyse ou de critique. D'après Valverde et Weir, il existe d'autres modes de régulation que celui de l'État. Le monde vécu et les traditions, les organisations volontaires et les institutions religieuses constituent autant de barrières à la régulation étatique. Cette critique souligne l'importance de l'étude empirique de l'étendue des divers modes de régulation sociale. Dénombrer le personnel de l'État revient à identifier les gens engagés dans le mode de régulation étatique ${ }^{7}$.

Le Canada-Uni connaît un développement remarquable de son système étatique et une extension importante de sa régulation. Mais, ces processus se déroulent dans un contexte où les modes de régulation alternatifs et concurrentiels sont particulièrement forts. La propriété féodale et son système patriarcal, le pouvoir croissant de l'Église dans le Canada-Est, la dominance des oligarchies locales et de nombreux groupements communautaires dans le Canada-Ouest constituent de vrais obstacles à la création d'un système d'éducation publique, à la reconnaissance de l'égalité entre propriétaires, à la régularisation des activités économiques ou à la promotion du projet bourgeois d' «hygiène sociale». D'autres grands projets de réglementation étatique rencontrent également des résistances: on refuse le système de gouvernement municipal dans le Canada-Est (au moins jusqu'en 1855); par crainte des taxes, on fausse délibérément les déclarations aux agents de recensement, etc. Néanmoins, une partie croissante de la vie sociale et économique se déroule à l'intérieur d'une réglementation étatique ${ }^{8}$.

7. Mariana Valverde et Lorna Weir, «Struggles of the Immoral», Resources for Feminist Research (automne 1988); aussi, Mariana Valverde, «Representing Childhood: the Multiple Fathers of the Dionne Quintuplets», C. Smart, ed., Regulating Womanhood: Historical Essays on Marriage, Motherhood, and Sexuality (London, Routledge, 1992), 119-146; The Age of Light, Soap, and Water: Moral Reform in English Canada, 1885-1925 (Toronto, McClelland and Stewart, 1991); "When the Mother of the Race is Free», F. Iacovetta et M. Valverde, eds., Gender Conflicts (Toronto, University of Toronto Press, 1992), 3-26.

8. Voir Bruce Curtis, «Révolution gouvernementale et savoir politique au Canada-Uni», Sociologie et Sociétés, 24 (1992): 169-179. 
Partant de ces considérations, nous voulons évaluer l'étendue du système étatique et déterminer sa place dans l'ensemble des rapports sociaux au Canada-Uni. Un premier pas serait de dénombrer les effectifs du state system. Mais nous aimerions cerner d'abord les embûches auxquelles se heurte la tentative d'utiliser les documents parlementaires et gouvernementaux pour dénombrer les serviteurs de l'État. Cet effort va alors nous mener à tenter d'identifier les gens mobilisés par le state project.

\section{UN MODÈLE DE BUREAUCRATIE}

Nous affrontons au point de départ un problème d'ordre conceptuel: les dénombrements officiels des travailleurs de l'État se contredisent. Si nous n'utilisons qu'une seule source officielle, nous trouvons d'une année à l'autre, des définitions discordantes du personnel de l'État; entre les différentes sources officielles de telles disparités sont encore plus marquées. En outre, les définitions officielles sont plus axées sur des considérations fiscales (qui est payé par le gouvernement?) que sur des considérations fonctionnelles (qui agit au nom de l'État?). Ainsi, nous ne pouvons pas emprunter naïvement les définitions de la bureaucratie offertes par les sources officielles. Pourtant, nous ne saurions identifier le personnel de l'État sans un modèle de sa bureaucratie, ou sans critères de distinction entre les travailleurs de l'État et les membres de la société civile.

La conception de la bureaucratie proposée par Max Weber pourrait nous être utile. Dans son modèle, les salariés ont des domaines de compétence délimités à l'intérieur d'une hiérarchie d'autorité. La vie privée des fonctionnaires est clairement séparée de leur vie professionnelle. Ils n'ont pas la propriété des documents et des rapports qu'ils produisent. L'accès aux postes est réglementé par un système d'examens écrits et non par des liens de parenté ou d'autres critères. L'emploi est permanent avec pension de retraite. Les fonctionnaires appliquent des règles de caractère universel aux cas spécifiques et, ayant acquis un sentiment de loyauté envers l'organisation, ils suivent les ordres des supérieurs sans égard à leur contenu'

Ce modèle de Weber aide, dans une certaine mesure, à comprendre la situation du Canada-Uni où quatre processus se développent simultanément: le nombre des membres du state system s'accroît; dans plusieurs secteurs, leurs activités se trouvent de plus en plus encadrées par des règles bureaucratiques; les façons de

9. Voir Max Weber, Économie et société (Paris, Plon, 1971), 219-252. 
représenter publiquement la bureaucratie se régularisent; et une mentalité vraiment bureaucratique se manifeste progressivement dans l'identité personnelle et dans les orientations sociales des hommes qui accèdent aux fonctions importantes du service de l'État ${ }^{10}$.

Ces développements ne sont ni linéaires, ni uni-directionnels et ne suivent aucun plan directeur. La création du bureau de vérification en 1855, par exemple, entraîne une réduction importante de la capacité des ministres de pratiquer le patronage par la création de nouveaux bureaux, de même qu'elle conduit à la régularisation des pratiques de la comptabilité. Mais de telles conséquences ne semblent pas avoir été voulues par les organisateurs de ce bureau. Les ministres impliqués semblent avoir simplement réagi au retrait du gouvernement impérial du financement de certains domaines (par exemple le département des Indiens), à la taille et à la croissance de la dette publique, de même qu'à certaines énigmes relatives aux dépenses des ministères ou départements ${ }^{11}$.

Les hommes politiques et leurs adjoints ont tenté avec plus ou moins d'énergie selon les conjonctures, de discipliner les fonctionnaires. La publication du célèbre rapport anglais Trevelyan-Northcote, prônant une bureaucratie d'experts, a soulevé un grand enthousiasme au Canada-Uni, mais les tentatives d'incorporer ses recommandations dans la pratique courante des bureaux ont souvent échoué. Pourtant, au moment de la Confédération, les procédures administratives témoignent d'un plus grand formalisme qu'en 1840.

Si l'orbite étatique s'étend dans certaines directions, elle se rétrécit dans d'autres. La formation de l'État ne se résume pas à une série d'interventions claires et nettes qui feraient gonfler la bureaucratie. Au contraire, il s'agit souvent de fonctions situées de plus en plus, ou de moins en moins, directement dans l'orbite étatique. Dans les années 1840, par exemple, les juges des districts du Canada-Ouest se voient imposer des qualifications d'avocat. Puis, au cours des années 1850, au lieu de se rétribuer à même les frais de justice, ils deviennent des

10. Weber insiste sur le fait que la supériorité technique de la bureaucratie comme mode d'administration réside partiellement dans sa durabilité acquise par le truchement du développement d'habitudes chez les fonctionnaires. Dans le cas du Canada-Uni, cette mentalité bureaucratique est très bien personnifiée par Andrew Russell du département des terres de la couronne, par le vérificateur, John Langton, ou par le ministre-adjoint de l'agriculture, JosephCharles Taché.

11. Voir W. A. Langton, ed., Early Days in Upper Canada. Letters of John Langton from the Backwoods of Upper Canada and the Audit Office of the Province of Canada (Toronto, Macmillan, 1926), 239-240. Le département de l'éducation du Canada-Est, donnait l'impression d'économiser, mais dépassait en fait son allocation budgétaire. Langton compare avec humour les pratiques financières de Ryerson et de Chauveau. 
agents salariés du gouvernement, lequel centralise et administre les amendes et les frais qu'ils imposent. Par contre, à la suite de la sécularisation des terres réservées au clergé dans le Canada-Ouest en 1855, les salaires des clercs sont commués en annuités. Les ministres anglicans et presbytériens partout au Canada-Uni, et les catholiques dans le Canada-Ouest, auparavant subventionnés directement par le gouvernement, cessent de l'être. L'appareil judiciaire devient ainsi plus directement un appareil d'État; l'appareil religieux l'est moins directement.

Nous remarquons un développement très inégal de la rationalisation bureaucratique dans différents secteurs administratifs. Déjà, au début des années 1820 , le Colonial Office avait ordonné à ses gouverneurs de ne pas considérer la correspondance officielle comme leur propriété personnelle. À la fin des années 1840 , le bureau de l'éducation du Canada-Ouest cherche à définir les documents émanant des inspecteurs d'école comme une propriété publique. Pourtant, jusqu'en 1855, sinon plus tard, les opinions juridiques officielles de l'avocat général et du procureur général sont traitées comme leur propriété personnelle. En quittant le gouvernement, ils emportent avec eux ces avis qui constituent néanmoins des pièces officielles. Au milieu des années 1860, les fonctionnaires municipaux quittent encore leur poste, documents officiels sous le bras ${ }^{12}$.

Les ministres et sous-ministres tentent périodiquement d'obliger leurs employés à adopter des habitudes d'assiduité, de ponctualité et de régularité. Mais, avant que le gouvernement n'ait acquis une identité architecturale bien définie dans ses bâtiments à Ottawa (après le mois d'octobre 1865), ses départements se trouvent éparpillés un peu partout dans des maisons de Kingston, Montréal, Toronto ou Québec. Les fonctionnaires subalternes, comme les ménagères et les messagers, vivent dans ces maisons; le travail d'administration se fait dans un cadre domestique, qui rend plus difficile l'établissement de normes bureaucratiques. Bref, la distinction commence à apparaître entre la vie publique et la vie privée, entre les activités officielles et les activités personnelles, entre l'État et la société civile, mais reste encore instable et floue.

La représentation des activités de l'État dans les documents officielles tout comme la comptabilité, sont de plus en plus normalisées. À plusieurs reprises, le Parlement exige des rapports départementaux dans lesquels le poste, la tâche et le salaire de chaque employé doivent

12. Voir ANC, RG1 E7, vol. 59A; B. Curtis, True Government by Choice Men? Inspection Education and State Formation in Canada West (Toronto, University of Toronto Press, 1992), chapitre 5; «Social Science and State Capacities: the Canada Blue Books», texte inédit, département de sociologie et d'anthropologie, Université Wilfrid Laurier, 1992. 
être clairement indiqués. L'État impérial fait des demandes semblables. Les contrôles exercés sur les dépenses se multiplient, surtout après 1855 . Les ministres se trouvent progressivement dans l'obligation de justifier la création d'emplois dans leur département et de détailler leurs dépenses. Par contre, même après la mise en place du bureau de vérification, les employés des ministères ne sont pas toujours identifiés dans les listes de mandats émis. La simple notation de paiements globaux attribués aux ministres «pour les salariés de leur département» refait surface de temps en temps, ce qui laisse survivre la pratique du patronage au sein des ministères.

En somme, la bureaucratie ne jaillit pas toute constituée de l'acte d'Union, mais résulte d'une dynamique historique. Ainsi, dans l'identification et le dénombrement des travailleurs de l'État, la difficulté est de situer les limites d'un processus qui change de taille, de forme et d'image.

\section{ON NE PEUT SE LIMITER À COMPTER DES NOMS}

Les principes bureaucratiques qui identifient les domaines de compétence des fonctionnaires, imposent des tâches à chacun et évaluent leur rendement de façon régulière ne sont pas encore bien établis. On ne peut donc se contenter d'additionner les noms inscrits dans les documents officiels pour définir le personnel du state system. Il faut aussi analyser les fonctions administratives qui y correspondent.

On ne peut non plus se laisser uniquement guider par les listes de paie car, dès les années 1840 , le gouvernement fait un grand nombre de paiements à des individus sans fonction administrative: des retraités de l'appareil judiciaire et des veuves de guerre, des coroners, des gardiens de prison et des gardiens de phares, des médecins en service dans les prisons et de nombreux autres individus aux fonctions moins importantes. Par contre, l'exécution d'une même fonction administrative ne rapporte pas toujours un salaire. Ainsi, tous les douaniers bénéficient du tiers du produit de leurs saisies, mais ceux de Prescott, Rondeau et Mariatown touchent en plus un salaire régulier, contrairement à ceux de Hemmingford, Huntingdon et Compton ${ }^{13}$. Bref, les listes de paie ne sont pas fiables pour dénombrer des bureaucrates.

Les rapports parlementaires et départementaux concernant le personnel de l'État sont des documents à forte teneur idéologique qui manifestent des intérêts politiques et administratifs particuliers. Ainsi,

13. Les premiers avaient un chiffre d'affaires plus important que les seconds; voir documents parlementaires, A, Appendice B.B. (1850). 
tel ou tel employé d'un département n'est pas inscrit sur les listes de la comptabilité parce qu'il n'en rencontre pas les critères. Par contre, la bureaucratie étant un lieu de patronage, des hommes sans charge ou fonction précise se retrouvent sur les listes de paie des départements. De plus, les frais contingents considérables attribués aux départements permettent parfois d'engager des fonctionnaires qui n'ont pas droit à un emploi régulier ou officiellement reconnu ${ }^{14}$. Enfin, il arrive que des retraités soient maintenus sur les listes de paie à titre de reconnaissance pour leurs loyaux services ou à la demande d'un ministre, car, malgré les efforts de P. N. Vankoughnet dans les années 1850, il n'y a pas avant la Confédération, de pension pour les fonctionnaires retraités.

Nous soupçonnons que le nombre d'hommes impliqués dans de telles pratiques est important. Quelques-uns sont assez faciles à identifier. Donald McLeod, par exemple, est nommé commis au bureau d'agriculture au mois de novembre 1853. À cette époque, trahi par sa main chancelante et ses incessantes fautes d'orthographe, McLeod a déjà passé l'âge du travail efficace. On lui accorde un congé de maladie au mois de juin 1855, congé qui se prolonge et devient une retraite avec pleine solde. Le bureau réduit sa solde vers 1860 , et un nouveau ministre de l'agriculture le congédie finalement en novembre 1862. Pendant plusieurs années, même s'il n'exerçe pas son métier, McLeod est présenté dans les sources officielles comme employé au bureau d'agriculture ${ }^{15}$.

Dans un autre cas encore plus frappant, le ministre des finances Luther Holton, à la suite de sa nomination en 1863, découvre que son sous-ministre, Joseph Cary, ne s'est pas présenté au bureau depuis presque huit ans. Pourtant, Cary reçoit régulièrement sa paie qui, vu son ancienneté, est très élévée. Le gouvernement lui a versé plus de $22000 \$$. Le conseil exécutif devait être au courant de l'affaire, car il avait autorisé un autre employé à faire le travail de Cary, advenant son

14. Chaque département se voit accorder ses propres frais contingents, et le département du sécretaire provincial en reçoit pour les besoins généraux du gouvernement. Ce montant d'argent est considérable. Pour l'année 1846, par exemple, T. D. Harington, le commis-en-chef au département, a reçu $6440 £$. En 1852-1853, le montant était $4950 £$. En cette année de recensement, les présidents du Conseil d'enregistrement et de statistiques en ont reçu pour $1800 £$ à eux seuls. Les employés subalternes gagnent alors de $75 £$ à $100 £$ par an. La totalité des frais contingents s'établissent à $74170,13 \$(18500 £$ et plus) en 1861 et à $60000 \$(15000 £)$ en 1866. Voir ANC RG19 vols. 1927; 2084-2085; 2094.

15. Sur McLeod, ANC, RG1 E7 vol. 51, 3 mars 1859; vol. 59, 3 novembre 1862; RG17 AI 2, Campbell à McLeod, 8 juin 1855; Hutton à Ross, 25 juillet 1860; Campbell à Reiffenstein, 10 février 1862; Campbell à McLeod, 6 novembre 1862; Campbell à L. Burwell, 14 septembre 1863. 
absence. Au même moment et dans le même département se trouve le commis J. R. Nash, payé pour un an à ne rien faire ${ }^{16}$.

Même en excluant les retraités, les absents et les inactifs dissimulés dans les listes de paie, il sera toujours difficile de faire une évaluation rigoureuse du nombre des fonctionnaires, car il y a la politique des frais contingents qui permet de cacher des engagements. Cette pratique est exposée et critiquée par Thomas D'Arcy McGee lors d'une étude des départements gouvernementaux en 1863. Le Civil Service Act de 1857 met en place un système d'examens semblable au modèle anglais pour réglementer l'accès aux emplois gouvernementaux. Mais, selon McGee, une personne qui a réussi les examens n'accède qu'à un emploi de fonctionnaire de quatrième classe, et doit attendre au moins deux ans avant d'obtenir une promotion. Par contre, prétend-il, une personne engagée à titre de «commis surnuméraire» n'a qu'à travailler dans un département pendant un mois pour faire régulariser par la suite son statut, sans avoir à commencer au bas de l'échelle. Cette voie d'accès est largement exploitée par les ministres et leurs adjoints, de sorte qu'il y a un nombre important d'employés surnuméraires qui ne paraissent ni dans la comptabilité officielle, ni sur les listes de paie. Cette situation donne lieu à des tentatives périodiques de contrôle de l'augmentation des frais contingents et de la régularisation du statut des employés «surnuméraires».

McGee propose donc l'abolition du Civil Service Act. Sa proposition reçoit peu d'écho et les abus dénoncés continuent de se produire. Par exemple, Joseph-Charles Taché n'accepte de devenir secrétaire du département d'agriculture, en 1864, qu'à la condition que son ami, l'abbé Cyprien Tanguay, obtienne un emploi régulier pour ses recherches généalogiques. Le salaire de Tanguay est d'abord tiré des frais contingents du département, et son nom ne figure que plus tard parmi les employés réguliers ${ }^{17}$.

Dénombrer les noms qui apparaissent sur les listes officielles n'est donc pas une méthode adéquate de recensement des travailleurs de l'État. Il faut aussi scruter attentivement l'administration des départements et les rôles du personnel pour identifier ceux qui ont un salaire sans occuper une fonction et ceux qui ont une fonction sans salaire.

\section{ON NE PEUT PROCÉDER PAR ÉCHANTILLONS}

Les initiatives inusitées, les événements inattendus, les changements de ministères et la conjoncture économique font croître ou

16. Sur Cary, ANC RG1 E7 vols. 62-63, 6 août 1863; sur Nash, 11 août 1863.

17. Sur McGee, ANC RG1 E7 vol. 59A; sur Taché, ANC RG17 AI 21864. 
diminuer le nombre de travailleurs de l'État. Par exemple, la réalisation des recensements de $1844,1848,1852$ et 1861 ajoute un nombre plus ou moins important d'employés pour des périodes allant de quelques mois à quelques années. Il s'agit en 1852 et en 1861 , d'environ quatre-vingt cinq commissaires, de quelques milliers d'agents de recensement et d'un nombre inconnu de commis affectés au bureau d'enregistrement et de statistiques ou au bureau d'agriculture. Ainsi, les $1800 £$ de frais contingents accordés au bureau d'enregistrement et de statistiques en 1852-1853, en montants de $100 £$ à $250 £$ par mois, auraient permis l'engagement d'un nombre important de commis, mais seul le secrétaire, unique employé permanent, paraît dans les sources officielles. Le secrétaire lui-même indique que son département engage un grand nombre de commis au jour le jour ${ }^{18}$.

Les épidémies (réelles ou anticipées) de choléra en 1849, 1854 et 1865 mènent à la création temporaire de bureaux de santé. La vague d'immigration irlandaise de 1847 impose aussi l'embauche de beaucoup de personnel. Par contre, il y a une réduction des employés de l'État en 1850, à la suite de l'accession au pouvoir des Réformateurs, une autre en 1857 occasionnée par la dépression économique, puis une autre en 1862 après l'avènement d'un nouveau ministère et à la suite d'enquêtes parlementaires sur l'immigration ${ }^{19}$. Ces faits suggèrent donc d'éviter d'évaluer la croissance du nombre de fonctionnaires par simple échantillonnage.

De plus, des chevauchements de juridiction entre les départements peuvent mener à compter la même personne comme fonctionnaire dans deux départements différents. Ainsi l'administration des terres de la couronne et le bureau d'agriculture se partagent la responsabilité de la planification et de l'administration des chemins de colonisation dans le Canada-Ouest au cours des années 1850. Les mêmes conducteurs de chemins de colonisation apparaissent sur les deux listes des rapports annuels des ministres de l'agriculture et des terres de la

18. Voir ANC RG19 vol. 1927 et RG17 AI 2, Crofton à Morin, 28 mars 1853 (Morin à Cameron, 26 mars 1853). Dans cette correspondance, Walter Crofton, secrétaire du bureau d'enregistrement et de statistiques, révèle qu'il ne peut pas répondre précisément aux requêtes parlementaires pour identifier et dénombrer les employés de son bureau. Il a «some doubt as to the kind of return I ought to make, owing to the peculiar character of my office, when the clerks are employed solely for the completion of the Census - are paid by the day - and have been increased or decreased in number, from time to time, as circumstances appeared to require. In short I am the only permanent Officer». Aussi, Documents parlementaires, 1853, document 12 de la comptabilité publique, pour les noms des 85 commissaires de recensement pour 1852 .

19. Voir, par exemple, First and Second Reports of the Select Committee of the Legislative Assembly, Appointed to Enquired into the Public Income and Expenditure of the Province (Toronto, Lovell and Gibson, 1850). Sur les coupures du début des années soixante au bureau d'agriculture, voir ANC RG17 AI 2, Campbell à Parent 24 avril 1863. 
couronne. Il en est de même de l'administration de l'immigration et de la colonisation partagée entre deux départements.

L'autonomie croissante de l'État colonial impose un dernier défi à ceux qui tentent de cerner les contours de la bureaucratie. L'accession de l'État colonial aux responsabilités détenues auparavant par l'État impérial oblige à définir précisément le point de départ. Doit-on compter au nombre des fonctionnaires tous ceux qui sont chargés de fonctions publiques pour la colonie, quelque soit leur lieu de travail, ou ne retenir que les fonctionnaires travaillant au Canada? Pour choisir la première perspective, il faudrait imaginer un moyen de séparer l'État colonial de l'État impérial, car il ne serait guère utile, par exemple, d'inclure parmi les serviteurs de l'État colonial tous les employés des postes en Angleterre avant 1851. Pour adopter la seconde, il faudrait suivre de près les changements de juridiction.

\section{UNE CARRIÈRE ET SA REPRÉSENTATION OFFICIELLE}

Compte tenu de ce qui précède, il nous paraît instructif de suivre la carrière d'un serviteur de l'État à travers les sources officielles. Celle de Walter Cavendish Crofton est particulièrement intéressante. $\mathrm{Ne}$ serait-ce qu'en raison de son rôle dans le développement de la statistique au Canada, Crofton mériterait l'étude approfondie qu'on attend toujours. C'est lui qui organise les recensements de 1848 et de 1852 et qui compile les données du rapport statistique annuel, appelé le Blue Book, de 1846 à 1854, sans parler de ses activités de généalogiste, héraldiste, cartographe, pamphlétaire, maître et inspecteur d'école et même de journaliste. Mais, dans la perspective méthodologique adoptée ici, le cas de Crofton nous intéresse surtout en raison du décalage entre la réalité de son emploi et sa représentation dans les sources officielles ${ }^{20}$.

Crofton, partisan de l'extrême droite, orangiste, entre dans la fonction publique avec l'avènement des Conservateurs en 1846. Il en est définitivement exclu en 1859, après des années de ballottement

20. Crofton, né vers 1806, probablement en Irlande, arrive au Canada en 1835, ayant été nommé maître de la grammar school du District de Newcastle au Haut-Canada. Il tient ce poste jusqu'en 1838, alors qu'il devient éditeur du Cobourg Star, journal d'allégeance ultraconservatrice. Il travaille pour la municipalité de Cobourg au début des années 1840 comme surintendant d'école, et cherche à entrer dans la fonction publique - comme surintendantadjoint de l'Éducation, par exemple. Il écrit des articles «statistiques» sur la région de Cobourg et offre ses services au public comme généalogiste. Il publie une carte du District de Newcastle, en plus d'articles et de pamphlets politiques. Plus tard, il tentera de faire subventionner un projet de manuel parlementaire. Crofton meurt à Toronto, où il avait terminé sa carrière comme clerc à Osgoode Hall, au mois de juillet 1870 . Malgré son statut du premier statisticien officiel du gouvernement canadien, Crofton reste inconnu. 
entre une fonction régulière et des postes officieux au gré des politiques partisanes. Son salaire ne figure que rarement dans les estimés gouvernementaux, et est plutôt tiré de fonds divers. Nous en déduisons que Crofton peut s'assurer des alliances assez fortes pour lui permettre de se nicher dans la bureaucratie étatique, mais pas assez fortes pour lui garantir un emploi stable. Par contre, l'instabilité de sa situation lui vaut d'être souvent nommé dans les rapports officiels ce qui facilite la reconstitution de sa carrière.

Crofton a trois tâches principales. De 1847 à 1855, il compile les données pour le Blue Book, l'une des entreprises statistiques les plus importantes de l'époque. Il planifie et surveille l'exécution des recensements de 1848 pour le Canada-Ouest et de 1852 pour les deux Canadas, en plus de préparer des sommaires statistiques. Ces tâches relèvent de son poste de secrétaire du bureau d'enregistrement et de statistiques. En plus, Crofton est officiellement engagé par le département de l'inspecteur général (devenu le département des finances en 1858) où il dresse le registre des billets de banque émis selon les lois sur les banques indépendantes.

La fonction de Crofton comme secrétaire du bureau d'enregistrement et de statistiques échappe aux coupures budgétaires consécutives à l'entrée en scène des Réformateurs en 1848, mais son avenir reste incertain. Il y a une autre raison à cela. Si les tableaux statistiques de la population qu'il a produits à la fin de 1848 lui valent l'approbation du gouverneur Elgin, celui-ci lui reproche son implication dans les émeutes entourant la loi pour l'indemnisation des victimes de 1837-1838. Elgin le décrit comme membre de la faction «ultra» des Tories, comme un homme dont il faut se méfier ${ }^{21}$.

Avec la création du bureau d'agriculture en 1852 et la surveillance du bureau d'enregistrement et de statistiques par le ministre de l'Agriculture, Malcolm Cameron, la situation de Crofton s'aggrave, car il avait déjà lourdement caricaturé Cameron dans un pamphlet publié sous le pseudonyme de «Erinensis ${ }^{22} »$. C'est peut-être à cause de son implication dans l'administration du recensement de 1852 que Crofton réussit à conserver sa position de secrétaire du bureau d'enregistrement et de statistiques jusqu'au 31 mars 1853. Il est alors

21. ANC RG1 E1, Canada State Book $H, 360,5$ avril 1848; MG24 A10, Elgin à Grey, 4 janvier 1849; Grey à Elgin, 17 janvier 1851; Elgin à Grey, 7 janvier 1851.

22. Voir «Erinensis» [Walter Cavendish Crofton], Sketches of the Thirteenth Parliament of Upper Canada (Toronto, Rogers and Thomson 1840), 16; aussi «Uncle Ben» [Walter Cavendish Crofton], A Brief Sketch of the Life of Charles, Baron Metcalfe... (Kingston, Athenaeum, 1846), pamphlet ultra-conservateur plein de sarcasmes aux dépens des Réformateurs. 
remplacé par William Hutton, un cousin du premier ministre Francis Hincks $^{23}$. Mais Crofton et Hutton sont tous deux payés à titre de secrétaire du bureau pendant le premier trimestre de 1853, et Crofton s'organise pour garder le contrôle de la préparation du Blue Book pour les années 1853-185424.

On suppose que Crofton retourne au sein du bureau de l'inspecteur général en 1853 , à la section de la vérification. William Cayley, inspecteur général lors de l'engagement de Crofton en 1846, redevient ministre en 1854 et il le restera jusqu'en 1858 . Il essaie, en 1855 , de placer Crofton de façon permanente au bureau de vérification, nouvellement créé. Cayley et le vérificateur John Langton (à l'insistance de Cayley) proposent d'accorder à Crofton un salaire régulier de $300 £^{25}$. Il semble que Crofton touche ce montant de 1855 à 1858. Il est finalement expulsé de la bureaucratie en 1859, après que A. T. Galt ait remplacé Cayley comme ministre des Finances. Galt ne voit aucune tâche qui puisse être remplie par Crofton au département des finances. Le gouvernement refuse le plaidoyer de ce dernier pour l'obtention d'un emploi régulier - en dépit de l'appui des députés et des conseillers conservateurs et des promesses qui auraient été faites à sa femme - et le congédie avec une compensation monétaire équivalant à neuf mois de salaire ${ }^{26}$.

Si nous essayons de retracer la carrière de Crofton à l'aide des sources officielles, les renseignements paraissent contradictoires, surtout vers la fin de sa carrière. Il y a cinq sources officielles faisant mention de Crofton: le Blue Book, la comptabilité publique, certains comptes rendus et autres rapports livrés au Parlement et publiés comme documents parlementaires, les listes de mandats émis par le receveur général et, à partir de 1857 , la main courante du vérificateur $^{27}$.

Dans le Blue Book qu'il a lui-même élaboré, Crofton apparaît comme employé au bureau de l'inspecteur général à titre de commis des statistiques, chargé de la compilation des données pour le Blue

23. Voir ANC MG30 E96 6, William Hutton à Anna Hutton Ponton, 16 et 17 mars 1853. Cameron dit attendre que Hincks trouve un poste à Crofton avant de nommer Hutton sécretaire du bureau d'enregistrement et de statistiques.

24. Sur l'histoire du Blue Book, voir Curtis, «Social Science and State Capacities: the Canada Blue Books».

25. ANC RG1 E7 vol. 42, 19 décembre 1855.

26. ANC RG1 E7 vol. 51, 3 mars 1859; vol. 52, 6 septembre 1859 (avec le plaidoyer). Ce sommaire est fondé non seulement sur les représentations officielles qui sont commentées plus bas, mais aussi sur les documents du cabinet et sur la correspondance départementale.

27. Nous ne faisons pas de distinction, pour le moment, entre les trois premières sources, qui ont été publiées, et les deux dernières, qui sont inédites. 
Book, et comme secrétaire du bureau d'enregistrement et de statistiques, jusqu'en 1853. Mais, après son renvoi du bureau, sa fonction y est moins clairement indiquée, et il disparait de ce rapport lorsque Hutton en prend charge.

La comptabilité publique donne une autre version de ces faits. Jusqu'en 1853, on mentionne de temps à autre que Crofton est secrétaire du bureau d'enregistrement et de statistiques et commis des statistiques chez l'inspecteur général. Cette source révèle une brève présence de Crofton au bureau d'agriculture en 1853, et indique indirectement son renvoi du secrétariat du bureau d'enregistrement et de statistiques. Crofton figure dans la comptabilité de 1855 parmi les commis à la section de la vérification au bureau de l'inspecteur général, mais en 1856, alors que les autres sources n'en disent rien, la comptabilité publique le place au bureau des postes. Cette source prétend que Crofton aurait travaillé comme commis au bureau d'agriculture pendant toute l'année $1857^{28}$ de même qu'en 1858 , alors qu'en 1859 il reçoit une prime de séparation de ce bureau ${ }^{29}$.

Nous trouvons également des traces de la carrière de Crofton dans divers documents parlementaires. Dans un rapport de 1849, il signe comme secrétaire du bureau d'enregistrement et de statistiques. En 1850,1855 et encore en 1856, les députés font publier la liste des bureaucrates avec une description de leurs tâches et de leurs salaires. Dans la liste de 1850, Crofton est placé à la section de la douane du département de l'inspecteur général, et on peut lire qu'il est «clerc des statistiques, et [qu']il tient le Blue Book». Dans la liste de 1855, Crofton est présenté comme compilateur des données du Blue Book et comme signataire des billets de la Bank of British North America au département de l'inspecteur général. Un rapport paru en 1856 prétend que Crofton a travaillé à la section du vérificateur du département de l'inspecteur général en $1855^{30}$.

28. ANC RG19 vol. 1930. Nous supposons que le commis chargé de la compilation de ces listes n'a regardé que les mandats pour le quatrième trimestre de l'année. Par contre, dans la main courante du vérificateur, Crofton figure comme employé au bureau pour toute l'année.

29. ALC, Documents parlementaires, appendice C, 1846; appendice A, 1847; appendice A, 1849 (pour 1847 et 1848); appendice C, 1850; appendice B, 1852-1853; appendice D, 18541855 ; appendice 30,1856 ; appendice 4,1857 ; appendice 4,1858 ; appendice 5,1859 ; appendice 1,1860 . Il est à noter que la comptabilité publique reproduit les listes de mandats émis; ces deux sources devraient être identiques; elles ne le sont pas.

30. First and Second Reports of the Select Committee of the Legislative Assembly, Appointed to Enquire into the Public Income and Expenditure of the Province (Toronto, Lovell and Gibson 1850); ALC, Documents parlementaires, appendice B, 1849, «Board of Registration and Statistics. Appendix to First Report»; appendice SSS, 1854-1855; son salaire est alors de $275 f$, en plus d'un montant de $40 £$ alloué pour ses frais, à partir du premier janvier 1853 . Il aurait reçu 590£ pour les années 1853-1854; appendice 60, 1856. 
Nous pouvons aussi retracer la carrière de Crofton à l'aide des listes de mandats émis par le receveur général ${ }^{31}$. La place et la fonction de Crofton y sont bien définies jusqu'en 1853, moment où il perd le poste du secrétaire du bureau d'enregistrement et de statistiques. Jusqu'alors, il est payé $200 £$ par an comme commis à la section de la douane du département de l'inspecteur général, et $75 £$ par an comme secrétaire du bureau. Son mandat de secrétaire se termine à la fin de mars 1853.

Ensuite, à part de brefs aperçus, nous le perdons de vue. Son nom ne figure sur aucune liste de paie départementale en 1854, 1855 ou 1856. La seule trace de son emploi est une prime de salaire qui lui est accordée comme commis en 1854, et une prime de déménagement allouée en 1856. Il ne paraît aucunement en 1855, année où il aurait travaillé chez le vérificateur. Mais, ce dernier a reçu un montant global pour payer ses employés, parmi lesquels Crofton aurait pu figurer.

Pendant les six premiers mois de 1857, Crofton est payé pour la tâche mal définie "[d'] employé au gouvernement civil», sans être attaché à aucun département; pour les six derniers mois de la même année, on le décrit comme commis au bureau d'agriculture et de statistiques. Le seul paiement effectué à son compte en 1858 est de $100 \$$ pour le mois d'octobre comme commis au même bureau. Pour les années 1855-1858, le salaire de Crofton se dissimule dans les interstices des pratiques comptables. Finalement en 1859, Crofton reçoit deux primes de séparation, l'une est accompagnée d'une note l'avertissant de ne plus se considérer comme appartenant à la fonction publique $^{32}$.

Nous trouvons une dernière version de la phase finale de la carrière de Crofton dans la main courante du vérificateur. Pour les années 1857-1858, il reçoit $300 £$ de salaire comme commis au bureau d'agriculture et statistiques. Pourtant, son nom n'apparaît pas sur la liste régulière des paiements aux employés de ce bureau. Crofton semble avoir reçu sa paie en vertu d'un mandat spécial ${ }^{33}$.

31. En principe, tous les paiements gouvernementaux se font par mandat, et tout mandat émis est numéroté et ses détails notés dans un livre spécial. La collection des mandats est incomplète. Dans l'incendie parlementaire de 1849, les livres de 1846 à 1848 ont été détruits. Quelques fois nous ne notons que des paiements globaux faits aux ministres pour payer leurs commis. Il y a égalemment d'autres lacunes dans la collection. Néanmoins, les listes de mandats permettent la reconstruction d'une grande partie de l'activité gouvernementale. À chaque déménagement du parlement, par exemple, on trouve des indemnités offertes aux fonctionnaires pour les frais de déménagement ou pour couvrir les dépenses d'un double loyer. On peut utiliser cette source de renseignements pour reconstituer des profils: qui a été payé? quand? combien? et pour quoi faire?

32. ANC RG19, vols 1926-1931.

33. Voir la main courante, ANC RG19, vols. 2078-2081. 
Les discordances des cinq versions de la carrière de Crofton sont frappantes. Nous ne saurions extraire des documents officiels une version crédible des activités de ce bureaucrate, malgré l'importance évidente de ses fonctions comme directeur des recensements et comme statisticien. Les contradictions des représentations officielles soulignent le caractère idéologique - sinon simplement fictif - du système documentaire. N'est-il pas étrange, par exemple, que Crofton soit payé comme commis au bureau d'agriculture et de statistiques pour les années 1857-1859 alors qu'il ne paraît ni dans les listes de paie ni dans le Blue Book préparés par le secrétaire de ce même bureau? Le secrétaire du bureau lui-même trouve cette situation outrageante! En effet, William Hutton observe dans un mémorandum envoyé au conseil exécutif au mois de février 1858:

Que dans la comptabilité publique pour l'année 1857, Schédule A, page 311, l'entrée suivante se présente.

W.C. Crofton - Salaire pour douze mois comme commis, bureau d'agriculture et de statistiques au 31 déc. 1857.....300.0.0

Que monsieur W. C. Crofton n'a jamais fait parti de ce Bureau, n'a jamais été lié à celui-ci d'aucune manière, - n'y a jamais rendu aucun service — n'a jamais été inscrit sur la liste de paie de ce Bureau - Qu'en conséquence, il ne peut y avoir de raison pour que son salaire puisse constituer une charge pour le département, comme il paraît présentement l'être... ${ }^{34}$

Au mois d'octobre de la même année, Hutton écrit à nouveau au sujet de Crofton, cette fois à P. N. Vankoughnet, remplaçant du ministre de l'Agriculture John Ross qui séjourne en Angleterre. Hutton précise avoir reçu de la part de Ross des instructions claires de ne reconnaître aucune relation entre Crofton et le bureau d'agriculture. Ross lui aurait dit: «Je n'endurerai aucune relation entre monsieur Crofton et ce bureau, d'aucune façon et pas même pour cinq minutes. Je le connais trop bien. Son nom ne sera pas associé au bureau, même pour recevoir sa paie.» Hutton cherche donc à faire payer Crofton directement par le conseil exécutif, mais apparement sans succès ${ }^{35}$.

Que faisait donc Crofton? Si les sources officielles nous laissent dans l'ignorance, deux lettres personnelles écrites par le vérificateur John Langton à son frère sont très révélatrices. Au moment où il prend charge du bureau de l'inspecteur général à la fin de 1855, Langton décrit ainsi la section de la vérification:

34. ANC RG1 E7, volume 51, 3 mars 1858. Traduction de l'auteur.

35. ANC RG17 AI 2, Hutton à Vankoughnet, 12 octobre 1858. Traduction de l'auteur. 
J'y ai trouvé trois commis... aucun d'entre eux ne vaut grandchose, mais il y en a deux qui sont assez «pratiques». Cependant, je rejette sur Cayley la responsabilité de m'avoir collé l'un des trois sur les bras. C'est un mouton noir qui a été ballotté de département en département et qu'on a trouvé partout tout à fait inutile - complètement incompétent et pire qu'incompétent. Cayley le savait aussi bien que moi, (...) J'ai protesté vigoureusement, car il reçoit le salaire le plus élévé de mon bureau et m'est complètement inutile. J'ai dit à Cayley que je n'ai rien contre le fait qu'il touche son salaire sans lever le petit doigt, mais je ne m'engage pas à lui trouver de l'ouvrage car je ne puis me fier à rien de ce qu'il fait. Je songe à m'en débarrasser bientôt...

Langton revient sur le sujet le 24 février 1856:

[L'un] qui touche $£ 300$ par an ne vaut pas grand-chose, mais je lui ai trouvé un travail sans relation avec les finances (car je ne puis compter sur lui quand il s'agit de chiffres) (...), bien qu'il soit une terrible peste, m'interrompant sans arrêt pour me dire ce qu'il a fait ou pour me consulter à propos de ce qu'il devrait faire. J'espère m'en débarrasser bientôt.

Langton ajoute qu'il donnera à un autre que ce commis la responsabilité de dresser les rapports des billets de banques. Évidemment, il s'agit de Crofton ${ }^{36}$.

Notre récapitulation des versions officielles de la carrière de Walter Crofton démontre qu'elles sont des constructions idéologiques plutôt que des relations fidèles de ses emplois. Il en ressort qu'il a fallu «faire quelque chose» avec Crofton, du moins aussi longtemps que son patron William Cayley restait sur la scène politique. Pour rendre compte de son emploi devant le Parlement, il était commode de le présenter comme appartenant au bureau de vérification en 1855, au bureau des postes en 1856 et au bureau d'agriculture de 1857 à 1859 . Si les représentations officielles donnent une image politiquement plus agréable que vraisemblable de la situation de Crofton, elles démontrent fort bien la fluidité des fonctions des travailleurs de l'État et la perméabilité de la bureaucratie. Elles montrent également que le dénombrement du personnel de l'État dans les sources officielles peut être trompeur.

36. W. A. Langton, ed. Early Days in Upper Canada. Letters of John Langton from the Backwoods of Upper Canada and the Audit Office of the Province of Canada (Toronto, Macmillan, 1928), 217-218; 231; traduction de l'auteur. Remarquons que Crofton, jugé incompétent «quand il s'agit de chiffres», a dirigé les recensements de 1848 et de 1852. 


\section{L'ÉTAT}

Les exemples précédents montrent éloquemment qu'un dénombrement rigoureux des travailleurs de l'État exige au départ une connaissance détaillée de la politique et des pratiques administratives de l'époque, même à l'intérieur des départements. Il faut d'abord faire la critique des sources officielles pour montrer comment les pratiques comptables mènent à des visions erronées du personnel. Il est nécessaire aussi de jumeler de façon systématique les rapports sur les effectifs et le travail exécuté à l'intérieur des bureaux. L'étude de la correspondance interne de chaque département et le recours aux sources biographiques permettent également de préciser la tâche des fonctionnaires et ainsi d'éliminer ceux qui sont payés selon les pratiques de patronage. Les chevauchements de juridiction entre les ministères et la double inscription d'une même personne peuvent être contournés en identifiant chacun des employés de l'État. Enfin, pour bien cerner le rythme de développement des effectifs bureaucratiques, il faut aussi repérer tous les événements susceptibles de perturber cette croissance: épidémies, crises économiques, enquêtes périodiques, transferts de juridiction entre l'État impérial et l'État colonial, etc. Mais même avec de telles précautions, il reste toujours la difficulté de distinguer le personnel de l'État des membres de la société civile. L'analyse critique de la documentation officielle laisse voir que les employés ne sont pas placés à des postes spécifiques dans une structure bureaucratique bien définie. Le modèle bureaucratique représente plus l'image officielle de l'organisation étatique qu'il ne cerne la réalité des initiatives et des activités des serviteurs de l'État.

Si le modèle bureaucratique ne permet pas d'identifier le personnel de l'État, où faut-il alors tracer la ligne qui sépare ce qui est l'État de ce qui ne l'est pas? L'État regroupe diverses initiatives et projets destinés à régler et à transformer la vie publique. Il en résulte qu'on ne peut pas distinguer nettement l'État de la société civile, ou la vie publique de la vie privée. Au mieux, de telles divisions seraient fluides et mouvantes, définies par les enjeux des politiques gouvernementales et des résistances des citoyens. Il ne faut donc pas considérer l'État comme un fait, un objet solide et invariable, un système doté d'une force de travail dont les membres sont identifiés par leur salaire.

Le rapport État-citoyen se pose en termes d'une dialectique d'assujettissement et de résistance, d'incorporation et de transformation, d'encadrement et de refus. Mais comment retracer ce rapport dans la réalité quotidienne? Pour dénombrer les gens mobilisés par les projets de l'État, il est à la fois nécessaire, et très difficile, de 
faire la distinction théorique État/non-État. La même difficulté se rencontre dans la pratique.

\section{L'ÉTAT ÉDUCATEUR}

Le cas de l'éducation publique, l'une des innovations majeures de la période de l'Union, peut illustrer cette difficulté. À cette époque, dans chacun des Canadas, un bureau d'éducation distinct est chargé de l'administration du système scolaire élémentaire. Le surintendant, aidé d'un petit groupe de commis, dirige des inspecteurs, des professeurs d'école normale, des membres des comités d'examen et des enseignants. Les écoliers, les contribuables et les membres des commissions scolaires sont également chargés d'obligations légales en matière d'éducation. De plus, dans le Canada-Ouest, des comités de comté s'ajoutent au comité provincial de l'instruction publique. Bref, l'éducation publique mobilise une foule d'individus. Qui donc fait partie de l'État? Où se situe la ligne de démarcation entre ce qui appartient à l'État en rnatière d'éducation publique et ce qui appartient à la société civile?

Encore une fois, les sources officielles sont discordantes. La comptabilité publique ne considère que le personnel du bureau d'éducation central. Or, au sein de ce personnel se trouvent les inspecteurs d'écoles du Canada-Est, mais non ceux du Canada-Ouest ${ }^{37}$. Il y a là un reflet des différences dans le financement de l'éducation, les inspecteurs étant payés par les municipalités dans le Canada-Ouest et par le bureau central dans le Canada-Est.

Les rapports des surintendants de l'éducation vont un peu plus loin. Dans le Canada-Ouest, par exemple, ils donnent les noms des inspecteurs et souvent, après 1855, ceux des maitres des grammar schools. Ces rapports indiquent le nombre d'instituteurs, mais ne les identifient pas. Omission fâcheuse car, dans une même année, un instituteur peut enseigner dans plusieurs écoles. Le corps enseignant est un corps d'État, même si la majeure partie de son salaire vient des municipalités et des parents. L'autonomie professionnelle n'existe pas: les matières enseignées et les pratiques pédagogiques sont définies par les administrations. Du reste, pour les instituteurs, qui sont tous obligés par les lois scolaires d'avoir une conduite exemplaire même à l'extérieur de l'école, la distinction entre la vie personnelle et la vie professionnelle n'est pas claire ${ }^{38}$.

37. Les rapports officiels mentionnent aussi les frais de l'École Normale de Toronto, mais sans donner le détail de leur composition.

38. Voir Bruce Curtis, Building the Educational State: Canada West, 1836-1871 (London, Althouse Press, 1988), chapitre 6; et True Government, 190. Avant l'Union de 1840, le Lower Canada Blue Book contient une liste des professeurs de la «Royal Institution»; voir, ANC, C.0.47/122, C.O.47/140 [bobines b-2903-b-2906]. 
Les lois de l'instruction publique attribuent des pouvoirs et des obligations à certains hommes respectables, comme les juges de paix, les conseillers municipaux ou les membres du clergé. Elles les nomment visiteurs des écoles avec le pouvoir, dans le Canada-Ouest par exemple, de tenir des assemblées pour soutenir les écoles et les bibliothèques de leur arrondissement. Ces mêmes lois imposent également le silence aux passants devant les écoles, défendent aux parents des écoliers d'intervenir dans la salle de classe et limitent la liberté des étudiants une fois entrés dans l'école.

Aucune des sources officielles ne nomme les membres des commissions scolaires ou, pour le Canada-Ouest, les membres des comités locaux de l'instruction publique. L'effet de ces exclusions est de taille. Limité au personnel des bureaux centraux, le projet éducatif ne regroupe qu'une vingtaine de personnes dans les années 1860; en ajoutant les inspecteurs, on atteint un peu plus de trois cent cinquante employés. Mais en fait, il y a plus de sept mille professeurs à la fin des années 1860 , et en général il y a trois commissaires pour chaque professeur, sans parler des membres des comités d'examen.

Est-il légitime ou raisonnable d'inclure les commissaires? Ils ne sont pas payés, mais ils ont des obligations légales face à l'administration de l'éducation. Ils ont un pouvoir réel, quoique limité, dans la conduite de l'instruction locale. Ils peuvent faire saisir les biens personnels pour recouvrer les taxes scolaires et signer des contrats avec les professeurs. S'il faut inclure les commissaires, logiquement il faut également compter d'autres fonctionnaires comme les évaluateurs de terre, les membres des conseils municipaux et les collecteurs de taxes. Autrement dit, pour cerner le plus précisément possible le personnel impliqué dans le projet éducatif, force est de remplacer le critère de paiement par l'État par celui d'activité dans le domaine de l'éducation. Bien que nécessaire, cette substitution mène à assimiler le state system au state project, à ne plus distinguer l'État de la société civile.

Considérée du point de vue du système d'administration, l'éducation publique relève d'une imbrication complexe des instances gouvernementales. Mais l'éducation publique est beaucoup plus qu'un système d'administration. En plus d'un regroupement de personnel, elle est conçue pour former et produire de loyaux sujets politiques. En tant qu'appareil idéologique, l'emprise du projet éducatif s'étend à tous les écoliers et à leurs parents, sinon à la société civile toute entière. À l'instar des projets de police ou d'hygiène publique, le projet éducatif vise à inculquer à la société civile des modèles de comportement par le truchement d'hommes et de femmes qui incarnent au sein des communautés un mode de régulation étatique ${ }^{39}$.

39. Sur la police, voir Carolyn Steedman, Policing the Victorian Community: the Organization of English Provincial Police Forces (London, Routledge and Kegan Paul, 1984), et The Radical Soldier's Tale (London, Routledge, 1990). 


\section{CONCLUSION}

Tout effort pour dénombrer les serviteurs de l'État, s'il ne se limite pas à une définition arbitraire de la bureaucratie (comme par exemple, le inside service, incluant seulement ceux qui travaillent à Ottawa), se bute inévitablement à la difficulté de démêler l'État et la société civile, surtout si l'État est interventionniste et cherche à inculquer des valeurs et à modeler des comportements. Il faut donc élargir notre conception des modes de régulation étatiques afin de ne pas confondre le pouvoir de l'État et l'organisation bureaucratique. Le modèle bureaucratique est peu apte à cerner ces initiatives de l'État. Il faut donc retracer les contours du state project, si l'on veut dénombrer rigoureusement le personnel de l'État. 